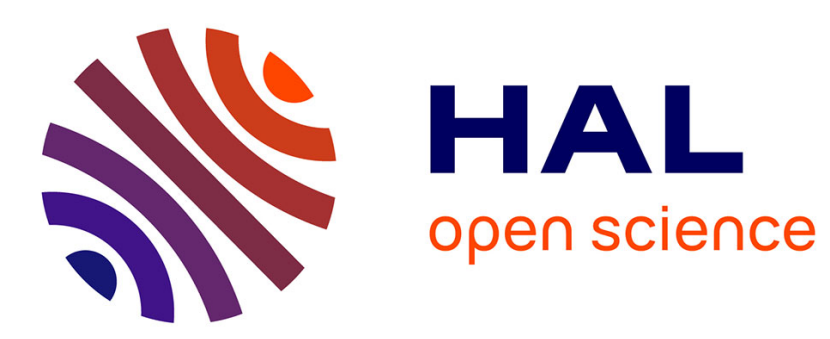

\title{
Quality control of CFRP by means of digital image processing and statistical point pattern analysis
}

\author{
D. Trias, R. García, J. Costa, N. Blanco, J.E. Hurtado
}

\section{To cite this version:}

D. Trias, R. García, J. Costa, N. Blanco, J.E. Hurtado. Quality control of CFRP by means of digital image processing and statistical point pattern analysis. Composites Science and Technology, 2007, 67 (11-12), pp.2438. 10.1016/j.compscitech.2006.12.023 . hal-00498980

\section{HAL Id: hal-00498980 \\ https://hal.science/hal-00498980}

Submitted on 9 Jul 2010

HAL is a multi-disciplinary open access archive for the deposit and dissemination of scientific research documents, whether they are published or not. The documents may come from teaching and research institutions in France or abroad, or from public or private research centers.
L'archive ouverte pluridisciplinaire HAL, est destinée au dépôt et à la diffusion de documents scientifiques de niveau recherche, publiés ou non, émanant des établissements d'enseignement et de recherche français ou étrangers, des laboratoires publics ou privés. 


\section{Accepted Manuscript}

Quality control of CFRP by means of digital image processing and statistical point pattern analysis

D. Trias, R. García, J. Costa, N. Blanco, J.E. Hurtado

PII: S0266-3538(07)00024-3

DOI: 10.1016/j.compscitech.2006.12.023

Reference:

CSTE 3567

To appear in:

Composites Science and Technology

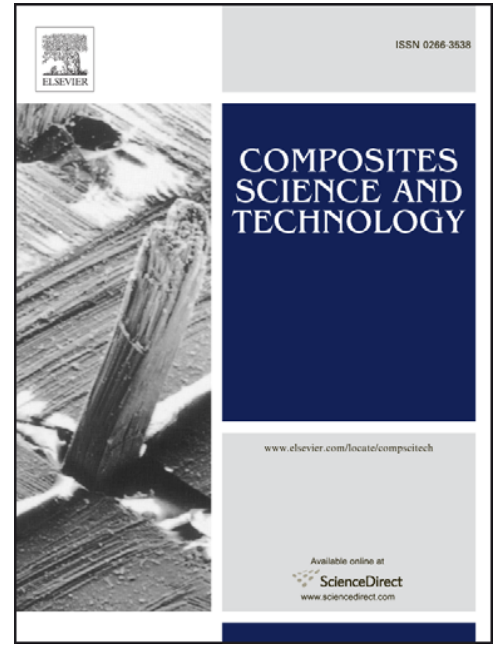

Received Date: $\quad 14$ September 2006

Revised Date: $\quad 22$ December 2006

Accepted Date: $\quad 23$ December 2006

Please cite this article as: Trias, D., García, R., Costa, J., Blanco, N., Hurtado, J.E., Quality control of CFRP by means of digital image processing and statistical point pattern analysis, Composites Science and Technology (2007), doi: 10.1016/j.compscitech.2006.12.023

This is a PDF file of an unedited manuscript that has been accepted for publication. As a service to our customers we are providing this early version of the manuscript. The manuscript will undergo copyediting, typesetting, and review of the resulting proof before it is published in its final form. Please note that during the production process errors may be discovered which could affect the content, and all legal disclaimers that apply to the journal pertain. 


\title{
Quality control of CFRP by means of digital image processing and statistical point pattern analysis
}

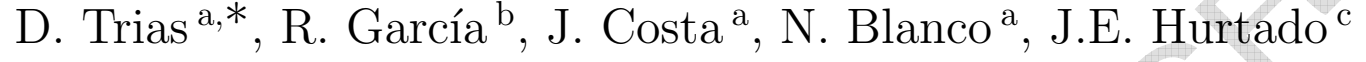 \\ a AMADE: Analysis of Advanced Materials for Structural Design. University of \\ Girona, Campus Montilivi s/n, 17071 Girona, Spain \\ ${ }^{\mathrm{b}}$ VICOROB: Computer Vision and Robotics Group. University of Girona, Campus \\ Montilivi s/n, 17071 Girona, Spain \\ ${ }^{\mathrm{c}}$ Universidad Nacional de Colombia, Sede Manizales. Apartado 12\%. Manizales, \\ Colombia
}

\begin{abstract}
Although fiber-reinforced composite materials have often been considered as periodic materials in theoretical models, the distribution of fibers is random in real materials. This random distribution of fibers is closely related to their transverse failure behavior. This paper proposes the use of statistical functions which describe random point patterns as a quantification of the dispersion of the transverse failure properties of several carbon fibre reinforced polymers (CFRP). It is shown that the analysis of the $\mathrm{K}$ function is the most meaningful for this purpose.
\end{abstract}

Key words: fiber-reinforced composites, optical microscopy, digital image processing, statistical properties, quality control 


\section{Introduction}

At the microscopical level, the main morphological characteristics of long fibre reinforced polymers are heterogeneity and anisotropy. In spite of this, composite materials have classically been modeled by means of periodical unit cells, that is, without taking into account neither the heterogeneity nor the geometrical disorder of fibers. The periodicity hypothesis leads to simplifications which make possible the application of homogenization methods [1-3], it provides good estimations for the elastic properties [4], and it can also be employed with good results in non-linear two-scale methods [5-9]. Also, in computational mechanics, the periodicity assumption leads to lower computational costs whereas other approaches may be computationally unaffordable.

However, a simple optical microscope observation reveals that long fiber reinforced composites (i.e. carbon or glass fiber-reinforced thermoset matrices) are far from being ordered materials since the fiber is randomly distributed through the matrix, sometimes showing areas with fiber clusters and resin pockets. These heterogeneities lead to local stress values in the matrix which are higher than those obtained assuming a periodical distribution and, consequently, they are more likely to produce damage, matrix cracking, or to cause degradation phenomena [10]. For this reason, the local damage in a transverse section of the composite (that is, matrix cracking and matrix-fiber debonding) is expected to depend strongly on the random distribution of the reinforcement.

On the other hand, because of the growing importance of composite materials

\footnotetext{
* Corresponding author

Email address: dani@emci.udg.es (D. Trias).
} 
in mechanical and structural engineering together with the lack of knowledge about many issues related to their failure, damage and fatigue behavior, there is a demand from the industry for quality control methods. This quality control methods should provide information on the defects within the material produced during manufacturing, the tolerance to these kind of defects, the relation between the material properties and its micro-scale structure. Traditionally, volume fraction is used as a measure of the quality of a laminate and ultrasound devices are normally used to complement this information by detecting voids and bubbles within the matrix.

Some researchers have proposed sophisticated and highly technological procedures such as thermal imaging techniques [11,12], optical coherence tomography [13], near-infrared spectroscopy $[14,15]$ or X-ray tomography [16] for the inspection of fiber-reinforced composites. Although these techniques are extremely precise, they usually require high technology machinery, sophisticated interpretation techniques and highly specified and qualified personnel. This makes them unusable for most industries.

The widespread use of computers in industry prompted some pioneering work, like that by Berryman [17], in data acquisition using digital image processing for heterogeneous materials. The quantitative techniques for digital image processing of composites are widely employed in metal matrix composites (MMCs) $[18,19]$ and some research has applied Fourier transformation to detect the orientation of reinforcement in reinforced concrete [20].

In fiber-reinforced polymers, much of the work devoted to the geometrical characterization of materials via digital image processing has been focused on braided composites [21]. Summerscales and co-workers computed total perime- 
ter and total area of inter-tow pore spaces in woven laminates produced by RTM $[22,23]$ and applied Voronoi tessellation and fractal dimensions to quantify the microstructure of woven composites [24]. The full characterization of glass and carbon fiber reinforced composites has also been addressed by means of optical microscopy [25] and digital image processing of micrographies has been employed by Joffe and Mattsson[26].

This work is part of a line of research which tries to bridge stress and strain fields at the macroscale with damage initiation and other microstructural phenomena by considering the random distribution of the fibers within the composite. This approach provides probability distribution functions for the stress and strain components, and is therefore useful for structural reliability pur-

poses. The methodology presented here starts from micrographies and, using image processing techniques together with spatial statistics tools, measures the homogeneity of the distribution of the fiber within the composite. Although the distribution of the fiber within the material is random, it is homogeneous - as will be shown in the next section the statistical homogeneity can be mathematically defined - if the fiber is correctly spread through out the material and, in this way, regions containing matrix pockets are avoided. This homogeneity can be seen as a measure of the quality of the fiber distribution since, as this paper will show, homogeneity in the material leads to lower mechanical property dispersion.

\section{Spatial point patterns}

This section summarizes the basics on spatial point patterns focusing on those aspects which are specially relevant for the statistical analysis of the 
microstructural characteristics of heterogeneous materials. Details on these topics can be found elsewhere [27-29].

A spatial pattern is a set of points which are located irregularly in a domain. The points' position is governed by some stochastic mechanism. Consider an image of area $A$ of a fiber-reinforced composite material, with the number $(N)$ and position $(\mathbf{x})$ of the center of the fibers being a random variable. Then, the set of the position of the centers is a spatial point pattern.

The first-order properties of a spatial point pattern can be described by the intensity function, $\lambda(\mathbf{x})$ :

$$
\lambda(\mathbf{x})=\lim _{|d x| \rightarrow 0}\left\{\frac{E[N(d x)]}{|d x|}\right\}
$$

where $E[\cdot]$ is the mathematical expectance operator. The second-order intensity function, $\lambda_{2}(\mathbf{x}, \mathbf{y})$, can be defined as:

$$
\lambda_{2}(\mathbf{x}, \mathbf{y})=\lim _{|d x|,|d y| \rightarrow 0}\left\{\frac{E[N(d x) N(d y)]}{|d x||d y|}\right\}
$$

which corresponds to the intensity function at $\mathbf{x}$ conditional on knowing that there is a fiber located at $\mathbf{y}$. The scaled function:

$$
g(\mathbf{x}, \mathbf{y})=\lambda(\mathbf{x}, \mathbf{y}) / \lambda^{2}
$$

is called the radial distribution function.

A usual assumption for the spatial point patterns found in heterogeneous ma- 
terials is that they are second-order stationary. This assumption implies that their statistical properties are invariant under translation, they have a constant mean, $\lambda((x))=\lambda$, and second-order properties can be expressed only as a function of the vector $\mathbf{r}=\mathbf{x}-\mathbf{y}$. Moreover, if a spatial point pattern can be considered isotropic, second order properties only depend on the modulus of vector $\mathbf{r}$, so we can write $\lambda_{2}(\mathbf{x}, \mathbf{y})=\lambda_{2}(r), g(\mathbf{r})=g(r)$. The point patterns given by the positions of fiber centres in fiber-reinforced composite materials can be considered isotropic second-order stationary and, consequently, the second order properties analyzed in this work only depend on $r$.

The usual probabilistic function which is assumed to describe the position of inclusions in a material is the Poisson point field $[28,29]$. This model describes complete spatial randomness (CSR) in the distribution of fibers. That means that the probability of finding $\mathrm{N}$ fibers in a subdomain of area $\mathrm{A}$ is the same for any chosen subdomain. Consequently, this model assumes that clusters of inclusions (fibers) do not take place.

The probability of finding $k$ fibers in a window $W$ of area $A(W)$ is given by:

$$
P[N=k]=\frac{(\lambda \cdot A(W))^{k}}{k !} \cdot e^{-\lambda \cdot A(W)} \quad k=0,1, \ldots
$$

where $\lambda$ is the fiber density, that is, the number of fibers per unit area.

Nevertheless, the Poisson distribution is physically unattainable due to finite dimension of the inclusions. For this reason, the Poisson distribution is 
often used for comparison purposes and may serve to distinguish between aggregated and more regular patterns. Therefore, a slight modification in the Poisson point field model is usually taken into account in order to reproduce real situations, in which the inclusions have a finite radius. In this case, the center of two inclusions cannot be closer than their diameters. So this model, which is also known in the literature as Matérn's model [28,29], is normally employed to describe the random position of the fibers within the composite. For the cases in which the radius of the fiber $r$ is much smaller than the sample window, it can be considered that the Poisson hard-core model and the Poisson model can be considered equivalent. This is the situation found in the materials described in this paper and, for this reason, only the Poisson distribution will be employed.

After these assumptions, the pair distribution function $g(r)$ can be defined as the probability of finding an inclusion whose center lies in an infinitesimal circular region of radius $\mathrm{d} r$ around the point $r$, provided that the coordinate system is located at the center of a second inclusion.

Another useful function for the statistical analysis of point patterns is Ripley's K-function, which can be defined as the number of further points expected to lie within a radial distance $r$ of an arbitrary point and divided by the number of points per unit area. Ripley's estimator [30] seems to be the most appropriate $[31]:$

$$
K(r)=\frac{A}{N^{2}} \sum_{k=1}^{N} w_{k}^{-1} I_{K}(r)
$$

where $N$ is the number of points in the observation area $A ; I_{K}(r)$ is the number of points lying within the circle of radius $r$ and with center located in 
the $\mathrm{k}$-th point; and $w_{k}$ is the proportion of the circumference contained within the sampling area $\mathrm{A}$ to the whole circumference with radius $r$. The weight $w_{k}$ can be computed numerically or using analytical expressions given in the literature [27].

[Figure 9 about here]

The following relation between $g(r)$ and $K(r)$ can be found [32]:

$$
g(r)=\frac{1}{2 \pi r} \frac{\mathrm{d} K(r)}{\mathrm{d} r}
$$

The second-order intensity function of a complete random pattern (CSR or Poisson set), $K_{P}(r)$, in a two dimensional domain is given by $[28,29]$ :

$$
K_{\mathrm{P}}(r)=\pi r^{2} \quad r>0
$$

Although $g(r)$ and $K(r)$ are related, they provide quite different physical information. $K(r)$ can distinguish different patterns and detect regularities, whereas the pair distribution function $g(r)$ describes the occurrence intensity of inter-inclusion distances. In this later function, a local maximum indicates the most frequent distances between points and a local minimum the least frequent ones in the pattern. The pair distribution function, , instead of the two-point probability function, can be used for the statistical description of a composite sample when the material can be considered ergodic and statistically isotropic.

The second order intensity function, $K(r)$, and the pair distribution function, 
$g(r)$, are useful to describe long-range interactions between points. Another interesting measure of how inclusions or fibers are distributed within the material is given by the nearest-neighbor distribution, which can be obtained easily as the probability distribution function of the shortest distance to a fiber for each fiber. Analogously, second- or third-nearest-neighbor distributions may be computed. These nearest neighbor functions focus on short-range interactions between points.

\section{Materials and techniques}

In this work, the analyzed composite materials were UD Laminates containing a nominal fiber volume fraction of 60\% (HTA 5131 400TEX 6K TO AERO Tenax). Three different matrices, RTM6 and 6376 from Hexcel and 977-2 epoxy resin from Cytec, were used. The composite with the RTM6 matrix was produced by resin transfer moulding and the others by a standard autoclave technique. All composites were cured at $180^{\circ} \mathrm{C}$ for $2 \mathrm{~h}$ and post cured at 190 ${ }^{\circ} \mathrm{C}$ for $4 \mathrm{~h}$. From the panel, standard tensile test specimens were cut according to DIN EN ISO 527-4, Type 3, and $45^{\circ}$ GFRP tabs were applied. Finally, for each material, seven specimens were tested in tension in the $90^{\circ}$ direction in a universal tensile machine (Zwick 1475) at a cross head speed of $1 \mathrm{~mm} / \mathrm{min}$. The elastic properties of HTA fibers and the different matrices can be found in Table 1. Results of tension tests are given in Table 2.

[Table 1 about here]

[Table 2 about here] 
The next step is the automatic detection of the fibers. In order to achieve this goal, image processing algorithms have been applied to the sequence of acquired images.

\section{Digital image processing techniques}

The automatic detection of the fibers should face two main challenges. Firstly, the radiometric properties of the acquired images prevent the fibers from being segmented from the matrix by a simple binarization. This radiometric artifact can be observed in the images as a non-uniform illumination field, brighter at the top-right corner of the image, and darker at the bottom-left corner. Figure 1 shows a sample image to be processed. Its radiometric properties can be observed by plotting the intensity component of the image as a depth-map, as shown in Figure 2. On the other hand, it is not possible to directly binarize the acquired images, since their histogram is not bimodal and, therefore, no unique threshold exists to correctly separate the pixels belonging to the fibers and from those of the matrix (see Figure 3). The second problem to be solved is the detection of the individual fibers in the segmented image. In view of the fact that a binary image segmenting the fibers presents a large number of connected fibers (e.g. see Figure 3a), it is not possible to directly apply standard image processing techniques -such as Connected Component Labeling (CCL) [33]- to label the fibers. On the other hand, we could consider using the a priori knowledge of the circular shape of the fibers. One alternative could be the use of the Hough transform [34]. Unfortunately, some of the fibers present radii that are quite different from the average (e.g. see Figure 4), generating a very large parameter space. The amount of possible radius in the parameter 
space, together with the lack of knowledge in the number of fibers to detect in every image, advises against the use of the Hough transform for circles.

[Figures 1, 2, 3 and 4 about here]

In this context, the proposed procedure to detect the fibers involves 2 phases:

( $i$ ) filtering and radiometric correction, and (ii) fiber segmentation and localization.

\subsection{Filtering and radiometric correction}

In order to carry out the radiometric correction of the images, the procedure illustrated in Figure 5 is applied.

[Figure 5 about here]

First, the image is divided into buckets. Every bucket has to be small enough to neglect local differences in the radiometric values inside the bucket, but big enough to ensure that a reasonable amount of pixels belonging to the two regions to be segmented (fiber and matrix) fall inside the bucket. Next, a local segmentation of the grey levels detected in every bucket is carried out. We should look for the two main regions, fiber and matrix, and compute the average value of the matrix grey levels. In some cases, a small third region, which belongs to the dust forming a deposit onto the sample, may appear . The pixels belonging to this third region can be observed in Figure $2 \mathrm{~b}$ as the vertical stripes at the lower bound of the depth map. It is expected that the amount of dirt will be very small when compared with the other two regions, and that it will have lower grey values. A fixed threshold is set to remove the 
pixels that belong to the dust (if any). The remaining pixels are segmented into the two regions (fiber and matrix), using the Otsu algorithm [35]. The local brightness-constancy assumption has proved to be valid for every bucket, enabling a correct segmentation of the matrix region. Then, the average value of the region corresponding to the matrix is computed for every bucket. Once a grid of mean grey level values is obtained, a second order 2D surface is fitted to them.

Finally, the original image is subtracted from the obtained surface. In this way, a corrected image presenting similar values for both fiber and matrix regions is obtained. The good performance of this procedure can be observed comparing the histograms of an original and a processed image (see Figure 6).

[Figure 6 about here]

\subsection{Fiber segmentation and localization}

Once the image has been radiometrically corrected, it presents a perfect bimodal histogram, as illustrated in Figure 6b. Therefore, a binarization can be carried out by choosing the correct threshold using the algorithm proposed by Otsu [35]. Then, a Connected Component Labeling (CCL) algorithm can be applied to the binary image [33]. Since the points in a connected component form a candidate region for representing an object, this algorithm finds the connected blobs in the image. After running CCL on the image, the algorithm assigns a unique label to all points in the same component. Our final aim is then to obtain a labeled image where every label belongs to an individual fiber in the image. Unfortunately, if we apply CCL to the binarized image, 
and then look for the largest connected component, the object illustrated with a bounding rectangle in Figure $7 \mathrm{a}$ is obtained. For this reason, further processing is required to segment the individual fibers. One way to "disconnect" objects that are linked together is to apply the erode operator as defined by the mathematical morphology [36]. This operator shrinks the objects by "eroding" their contour, but erosion of a binary image has the problem: the objects that are smaller than the size of the structuring element used for eroding are eliminated. A priori, this should not be a problem, since a small $3 \times 3$ structuring element is used. However, due to the nature of the images illustrated in this work, several iterations of erosion are required to "disconnect" individual fibers. For this reason, instead of applying the erosion to the whole image, the biggest connected component of the image is segmented from the rest of the image, and the erode operation is applied only to this element. This procedure produces an image where only this connected component is eroded, and the rest of the image remains unmodified (see Figure 7). Next, the biggest connected component of the object is located through CCL, and the segmentation/erosion procedure is repeated recursively until the biggest object is the size of an individual fiber. Finally, CCL provides a unique label for every fiber of the image. Then, computing the gravity center of every connected component provides the localization of the fibers, as shown in Figure 8. Provided that most image processing algorithms involving the use of neighborhood operations (i.e., applying structuring elements, masks, etc.) fail to correctly process the boundaries of the image, the outline of the image is ignored to obtain statistical data. This outline has been set to the radius of a standard fiber.

[Figures 7 and 8 about here] 


\section{$5 \quad$ Results and discussion}

For each of the materials described in Section 3, 40 images have been analyzed with the techniques just described. As a result, the position of the center of the fibers in each image has been determined.

\subsection{Volume fraction}

The first magnitude which can be easily computed from the acquired data is the fiber content $\left(v_{f}\right)$. This is a key magnitude which governs the composite behavior and from which elastic constants are usually estimated. Table 3 shows the mean $(\mu)$, variance $\left(\sigma^{2}\right)$ and coefficient of variation $(\rho)$ computed for each of the three analyzed materials. While HTA5131/RTM6 and HTA5131/977-2 have similar properties, clearly HTA5131/6376 has a higher mean and much lower values of variance and coefficient of variation.

[Table 3 about here]

\subsection{Second-order functions}

$K(h)$ and $g(h)$ (Equations 3 and 5, respectively) can be used to assess if a point pattern describes complete spatial randomness.

For each of the images of all three materials, functions $K(h)$ and $g(h)$ were

computed. Let us denote with $\tilde{K}_{i}^{(j)}(h)$ and $\tilde{g}_{i}^{(j)}(h)$ the estimations of functions $K(h)$ and $g(h)$ computed for the i-th image for the j-th material. Then, for 
each of the materials, we can analyze the statistics of $K(r)$ and $g(r)$ :

$$
\begin{aligned}
& \mu\left[K^{(j)}(r)\right] \simeq \tilde{K}^{(j)}(r)=\frac{1}{m} \sum_{i=1}^{m_{j}} \tilde{K}_{i}^{(j)}(h) \\
& \operatorname{Var}\left[\tilde{K}^{(j)}(r)\right] \simeq \frac{1}{m(m-1)} \sum_{i=1}^{m_{j}}\left\{\tilde{K}_{i}^{(j)}(r)-\tilde{K}^{(j)}(r)\right\} \\
& \rho\left[\tilde{K}^{(j)}(r)\right] \simeq \frac{\operatorname{Var}\left[\tilde{K}^{(j)}(r)\right]}{\tilde{K}^{(j)}(r)} \\
& \mu\left[g^{(j)}(r)\right] \simeq \tilde{g}^{(j)}(r)=\frac{1}{m} \sum_{i=1}^{m_{j}} \tilde{g}_{i}^{(j)}(r) \\
& \operatorname{Var}\left[\tilde{g}^{(j)}(r)\right] \simeq \frac{1}{m(m-1)} \sum_{i=1}^{m_{j}}\left\{\tilde{g}_{i}^{(j)}(r)-\tilde{g}^{(j)}(r)\right\} \\
& \rho\left[\tilde{g}^{(j)}(r)\right] \simeq \frac{\operatorname{Var}\left[\tilde{g}^{(j)}(r)\right]}{\tilde{g}^{(j)}(r)}
\end{aligned}
$$

Figures 10 and 11 show plots for these statistics. Figure 10 (top) shows $\mu[K(r)]$ for each material together with the plot of the analytical expression for a Poisson set (Equation 7). All four plots are nearly superposed, showing that, on average, all three materials could be considered to contain a CSR pattern for the distribution of fibers.

[Figure 10 about here]

[Figure 11 about here] 
A closer observation of the plots of $\tilde{K}^{(j)}(r)$ reveals that the superposition of the plots is more remarkable for $r / R<15$, where $r$ is the inter-fiber distance and $\mathrm{R}$ the fiber radius. For larger values of $r / R$ the plot for HTA5131/977-2 seems to depart slightly from the CSR plot. The difference with respect a CSR pattern can be analyzed by computing the relative error for each material $j$ :

$$
E\left[K^{(j)}(r)\right]=\frac{\tilde{K}^{(j)}(r)-K_{P}(r)}{K_{P}(r)}
$$

According to the plot for this function, given in Figure 12, the HTA5131/6376 material is clearly much closer to the CSR pattern than the others.

[Figure 12 about here]

The plots for the variance and coefficient of variation of $K(r)$, also given in Figure 10. As happened in the results for the volume fraction, given in Table 3, the variance found in HTA5131/6376 is much lower than in the other materials, .

Regarding Figure 11, analogous conclusions can be derived: all three materials seem to have a $g(r)$ function close to that corresponding to a CSR pattern and the variance and coefficient of variation is much lower for the HTA5131/6376 material. The relative difference with respect to a CSR pattern can be computed for $g(r)$ as was done for $K(r)$ in Equation 14. The resulting plots for $E\left[g^{(j)}(r)\right]$, shown in Figure 11, and the relative error, shown in Figure 13, are not as meaningful as they were for $E\left[K^{(j)}(r)\right]$.

[Figure 13 about here]

However, from the fiber distribution analysis and the statistics of volume frac- 
tion and from the results of tensile tests shown in Table 2 a clear conclusion can be drawn: those materials showing a large dispersion on tensile test also exhibit a large dispersion on volume fraction and a large dispersion from a CSR distribution. Analysis of the $\mathrm{K}$ function reveals this phenomenon more clearly than the analysis of the fiber volume fraction.

\section{Conclusions}

The statistical point patterns of fiber positions have been analyzed for three different carbon fiber-reinforced polymers. Data for the statistical analysis has been obtained through digital image processing: $(i)$ filtering and radiometric correction, and (ii) fiber segmentation and localization. In this way, 40 digital images for three different CFRPs have been analyzed. The volume fraction and the position of the fiber centers has been obtained for each digital image. Together with a statistical analysis of the volume fraction, the functions $K(r)$ and $g(r)$ which describe the random distribution of fibers within the material have been computed and compared with the respective functions for a Complete Spatial Random (CSR) pattern.

The results of this analysis reveal that the materials showing a distribution of fibers which is more different from a CSR pattern have a larger dispersion in their failure behavior,and the analysis of the $\mathrm{K}$ function is the most meaningful.

The tools presented could be easily employed in an industrial environment as quality control measurements, since the small dispersion in failure behavior is a desired property in mechanic engineering materials. 


\section{Acknowledgements}

The work of D. Trias, J. Costa and N. Blanco has been partially funded by the Spanish Government under research project MAT2003-09768-C03-001. The work of R. Garca has been partially funded by the Spanish Government under research project CTM2004-04205. D. Trias would like to express gratitude to the University of Girona for doctoral research grant BRAE00/02.

Digital images and tensile test results were provided for a former collaboration by Bodo Fiedler and Thomas Hobbiebrunken of the composites group the Technical University of Hamburg-Harburg (TUHH). 


\section{References}

[1] P. Suquet. Homogenization Techniques for Composite Media, chapter Elements of homogenization for inelastic solid mechanics, pages 193-279. Springer-Verlag, 1987.

[2] E. Sanchez-Palencia. Homogenization Techniques for Composite Media, chapter Boundary Layers and Edge Effects in Composites, pages 121-192. Springer Verlag, 1987.

[3] J. Sanchez-Hubert and E. Sanchez-Palencia. Introduction aux méthodes asymptotiques et à l’homogénéisation. Masson, 1992.

[4] C. T. Sun and R. S. Vaidya. Prediction of composite properties from a representative volume element. Composites Science and Technology, 56(2):171$179,1996$.

[5] J.C.Michel, H.Moulinec, and P.Suquet. Effective properties of composite materials with periodic microstructure: a computational approach. Computer Methods in Applied Mechanics and Engineering, 172(1-4):109-143, 1999.

[6] S. Li. General unit cells for micromechanical analyses of unidirectional composites. Composites Part A: Applied Science and Manufacturing, 32(6): $815-826,2000$.

[7] L. Huyse and M. A. Maes. Random field modeling of elastic properties using homogenization. Journal of Engineering Mechanics, 127(1):27-36, 2001.

[8] E. Car, F. Zalamea, S. Oller, J. Miquel, and E. Oñate. Numerical simulation of composite materials: two procedures. International Journal of Solids and Structures, 39(7):1967-1986, 2002.

[9] F. Feyel. A multilevel finite element method $\left(\mathrm{FE}^{2}\right)$ to describe the response of 
highly non-linear structures using generalized continua. Computer Methods in Applied Mechanics and Engineering, 192(28-30):3233-3244, 2003.

[10] D. Trias, J. Costa, J.A. Mayugo, and J.E. Hurtado. Random models versus periodic models for fibre reinforced composites. Computational Materials Science, 38(2):316324, 2006.

[11] D.P. Almond and W. Peng. Thermal imaging of composites. Journal of Microscopy, 201(2):163-170, 2001.

[12] C. Blanco, S. P. Appleyard, and B. Rand. Study of carbon fibres and carboncarbon composites by scanning thermal microscopy. Journal of Microscopy, 205 (1):21-32, 2002 .

[13] J. P. Dunkers, F. R. Phelan, D. P. Sanders, M. J. Everett, W. H. Green, D. L. Hunston, and R. S. Parnas. The application of optical coherence tomography to problems in polymer matrix composites. Optics and Lasers in Engineering, $35(3): 135-147,2001$.

[14] W. Li, Y.D. Huang, L. Liu, and N.T. Chen. Rapid and non-destructive analysis of quality of prepreg cloth by near-infrared spectroscopy. Composites Science and Technology, 65(11-12):1668-1674, 2005.

[15] G. Lachenal and Y. Ozaki. Advantages of near infrared spectroscopy for the analysis of polymers and composites. Macromolecular Symposia, 141:283-292, 1999.

[16] P.J. Schilling, B.R. Karedla, A.K. Tatiparthi, M.A. Verges, and P.D. Herrington. X-ray computed microtomography of internal damage in fibre reinforced polymer matrix composites. Composite Science and Technology, 65(14):20712078, 2005.

[17] J. G. Berryman. Measurement of spatial correlation functions using image processing techniques. Journal of Applied Physics, 57(7):2374-2384, 1985. 
[18] P.A. Karnezis, G. Durrant, and B. Cantor. Characterization of Reinforcement Distribution in Cast Al-Alloy/ $\mathrm{SiC}_{p}$ Composites. Materials Characterization, 40 (1):97-109, 1998.

[19] Y. D. Huang, L. Froyen, and M. Wevers. Quality control and nondestructive tests in metal matrix composites. Journal of Nondestructive Evaluation, 20(3): 113-132, 2001.

[20] C. Redon, L. Chermant, J.-L. Chermant, and M. Coster. Assessment of fibre orientation in reinforced concrete using Fourier image transform. Journal of Microscopy, 191(3):258-265, 1998.

[21] B. Lian, L. Jiang, J.J. McGrath, and J. Jaranson. Quantitative determination of morphological features of triaxially braided composites by the use of machine vision. Composites Science and Technology, 60(2):159-166, 2000.

[22] J. Summerscales, P. R. Griffin, and S. M. Grove. Quantitative microstructural examination of RTM fabrics designed for enhaced flow. Composite Structures, 32(1-4):519-529, 1995.

[23] N. R. L. Pearce, J. Summerscales, and F.J. Guild. The use of automated image analysis for the investigation of fabric architecture on the processing and properties of fibre-reinforced composites produced by RTM. Composites Part A: Applied Science and Manufacturing, 29(7):829-837, 1998.

[24] J. Summerscales, N.R.L. Pearce, P. Russell, and F.J. Guild. Voronoi cells, fractal dimensions and fibre composites. Journal of Microscopy, 201(2):153$162,2001$.

[25] N. C. Davidson, A. R. Clarke, and G. Archenhold. Large-area, high-resolution image analysis of composite materials. Journal of Microscopy, 185(2):233-242, 1997. 
[26] R. Joffe and D. Mattsson. Methodology for characterization of internal structure of NCF composite and its influence on mechanical properties. In 3rd International Conference on Composites Testing and Model Identification CompTest 2006, held on the 10th - 12th April 2006 hosted by the Faculty of Engineering, University of Porto, Portugal, 2006.

[27] P. J. Diggle. Statistical analysis of spatial point patterns. Arnold: London, 2003.

[28] J. Ohser and F. Mücklich. Statistical Analysis of Microstructures in Materials Science. Statistics in Practice. John Wiley and Sons, 2000.

[29] K. Sobczyk and D. J. Kirkner. Stochastic Modeling of Microstructures. Modeling and Simulation in Science, Engineering and Technology. Birkhäuser, 2001.

[30] B.D. Ripley. The second-order analysis of stationary point processes. Journal of Applied Probability, 13:255-266, 1976.

[31] S. Mucharreira de Azeredo Lopes. Statistical Analysis of Particle Distributions in Composite Materials. $\mathrm{PhD}$ thesis, University of Sheffield. Department of Probability and Statistics, 2000.

[32] R. Pyrz. Quantitative description of the microstructure of composites. part 1: Morphology of unidirectional composite systems. Composites Science and Technology, 50(2):197-208, 1994.

[33] F. Chang, C-J. Chen, and C-J. Lu. A linear-time component-labeling algorithm using contour tracing technique. Computer Vision and Image Understanding, 93(2):206-220, 2004.

[34] D.H. Ballard. Generalizing the Hough transform to detect arbitrary shapes. Pattern Recognition, 13(2):111-122, 1981.

[35] N. Otsu. A thresholding selection method from gray-level histogram. IEEE Transactions on Systems, Man, and Cybernetics, 9(1):62-66, 1979. 
[36] R. Gonzalez and R. Woods. Digital Image Processing, 2nd Edition. Prentice Hall, New Jersey, 2002. 


\section{List of Figures}

1 Sample image to be processed.

2 Two views of the depthmap representing the grey values of the image of Figure 1. (a) Orthogonal view. (b) Perspective view.

3 Result of applying a binarization to the original image according to Otsu's algorithm [35]. (a) Threshold is set to 0.65. Notice that it produces a poor segmentation at the top right and bottom-left corners. (b) Result of applying a 0.69 threshold to correctly segment the bottom-left fibers. It can be observed how a small change in the threshold produces a big change in the segmentation. This means that the selected threshold is located at a very unstable point.

4 Detail of 3 images showing fibers of different radii.

5 Bloc diagram of the radiometric correction procedure.

6 Histograms of (a) the original image illustrated in Figure 1, and (b) the image obtained after radiometric correction. In (a) it is impossible to find an adequate threshold, as illustrated in Figure 3. The (b) histogram is now well conditioned to binarize the image applying Otsu's algorithm. 
7 Binarization of the radiometrically corrected image. (a) The red rectangle frames the largest object after applying a Connected Component Labeling (CCL) strategy. The largest object defined in (a) is eroded, giving rise to image (b). Now the objects with maximum height (red) and width (blue) are framed.

8 Localization of the automatically detected fibers. Due to the nature of the algorithm, it can be observed how the algorithm fails to correctly locate the center of the fibers when they are touching the border of the image. For this reason, only the fibers having their gravity center within the rectangle are considered.

9 Estimation of Ripley's K-function, $K(h)$.

10 Mean(top), variance(middle) and coefficient of variation (bottom) of $K(h)$ for the analyzed materials

11 Mean(top), variance(middle) and coefficient of variation (bottom) of $g(h)$ for the analyzed materials 


\section{List of Tables}

1 Elastic properties of the fiber (HTA5131) and the three analyzed matrixes. (*) Transverse modulus

2 Ultimate strain statistics for three different CFRPs

$3 \quad$ Volume fraction statistics computed for three different CFRPs 43 


\section{Figures}

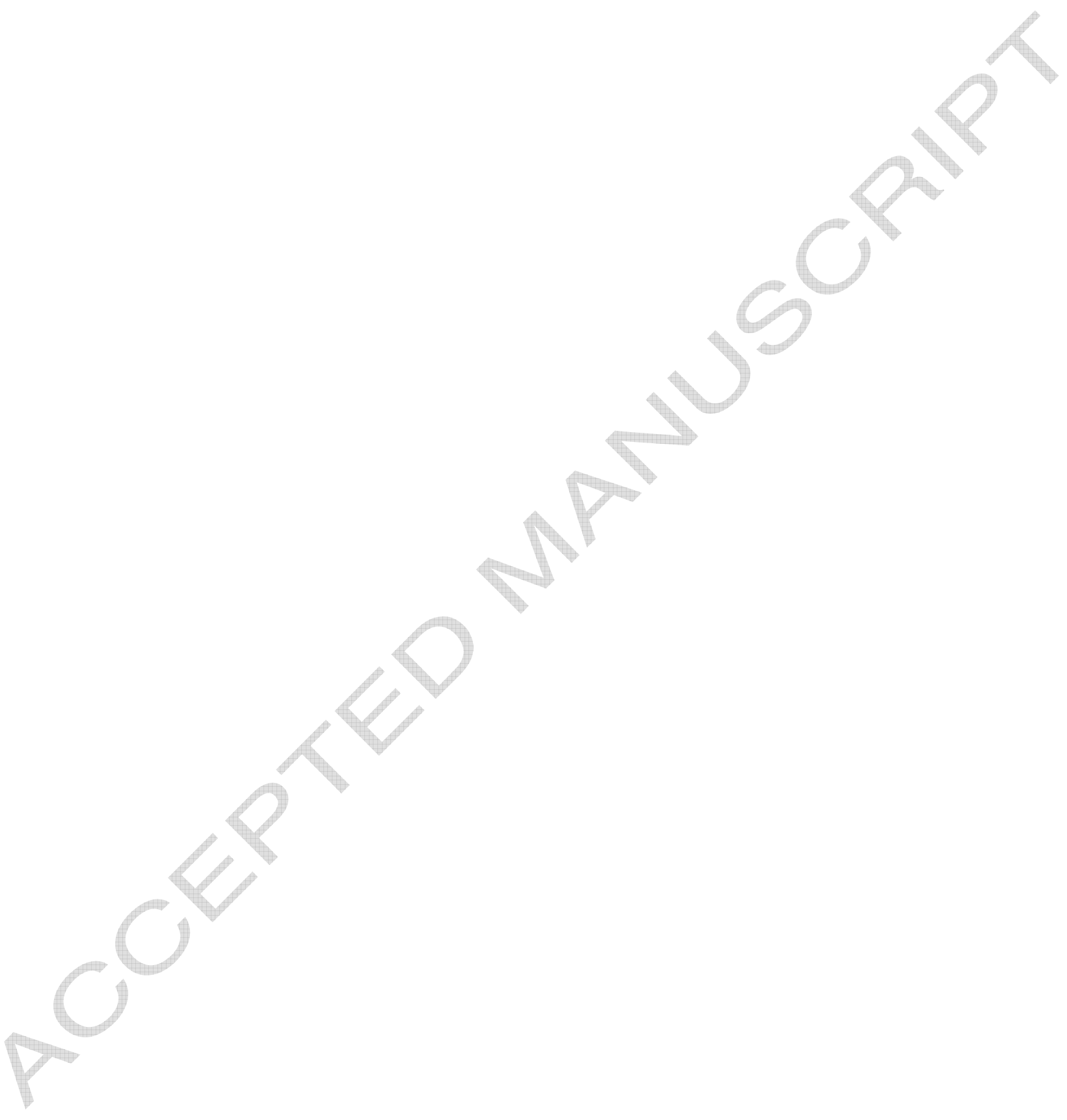




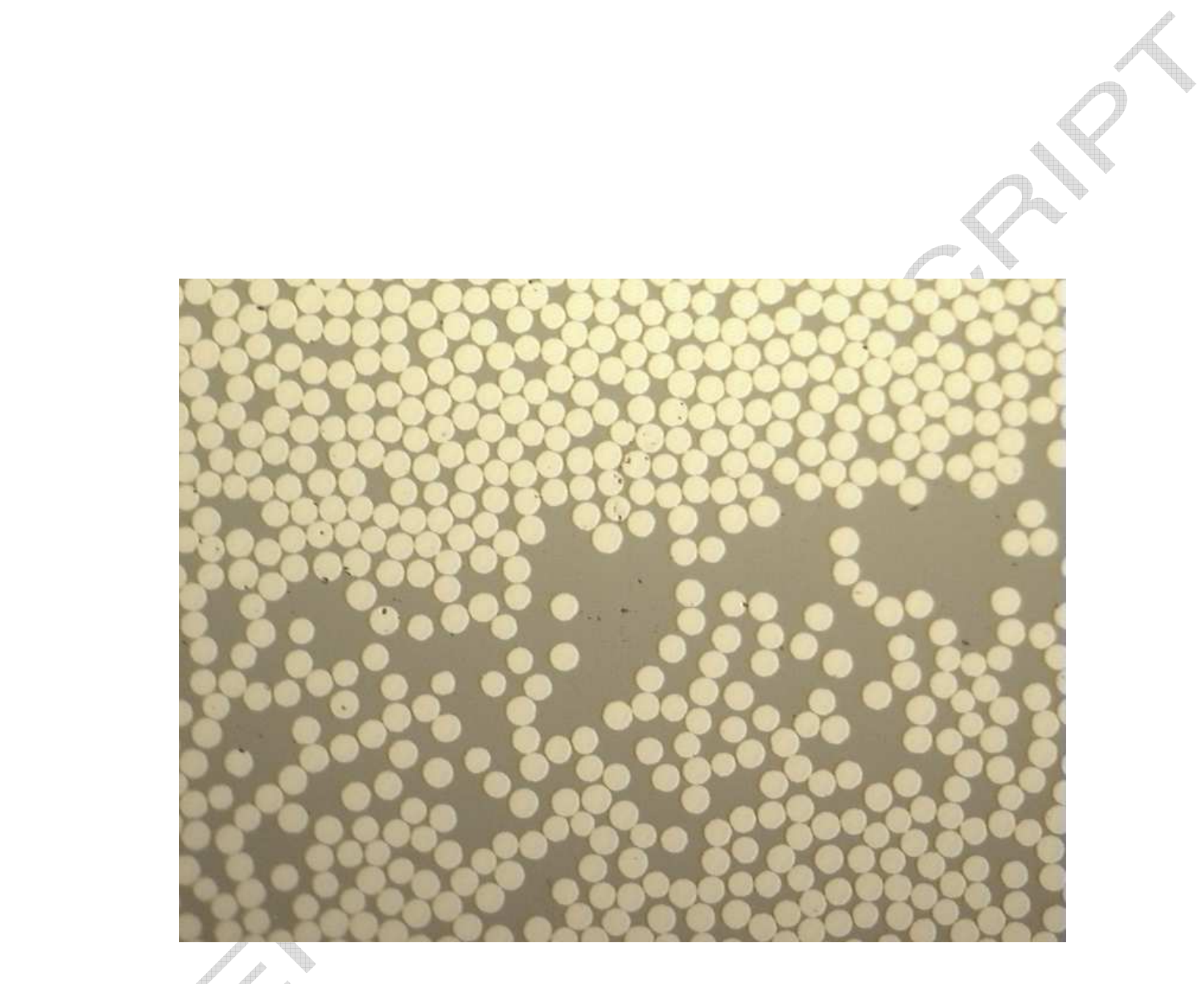

Fig. 1. Sample image to be processed. 


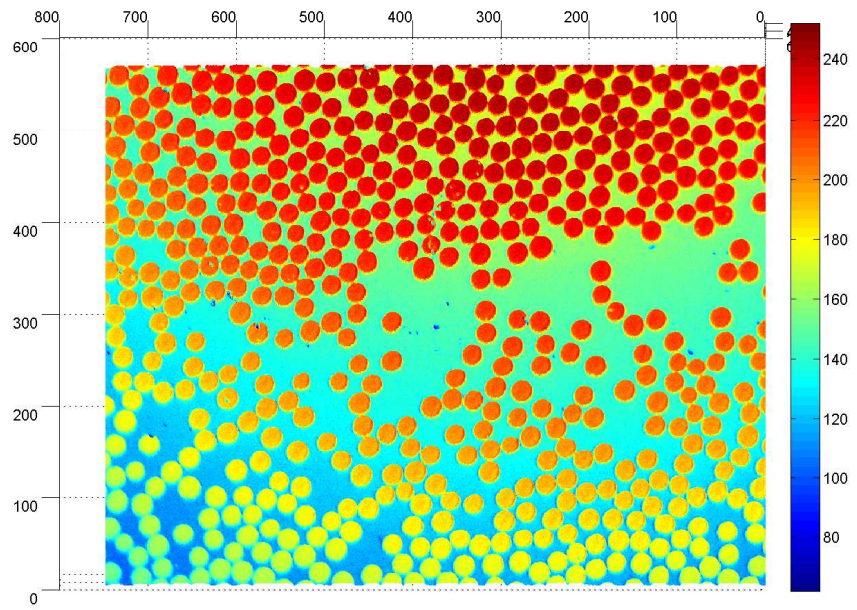

(a)

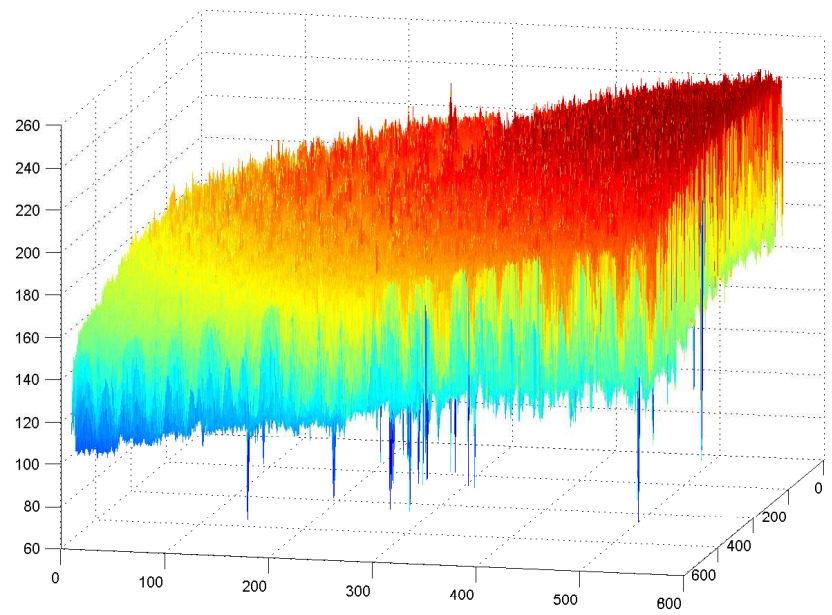

(b)

Fig. 2. Two views of the depthmap representing the grey values of the image of Figure 1. (a) Orthogonal view. (b) Perspective view. 


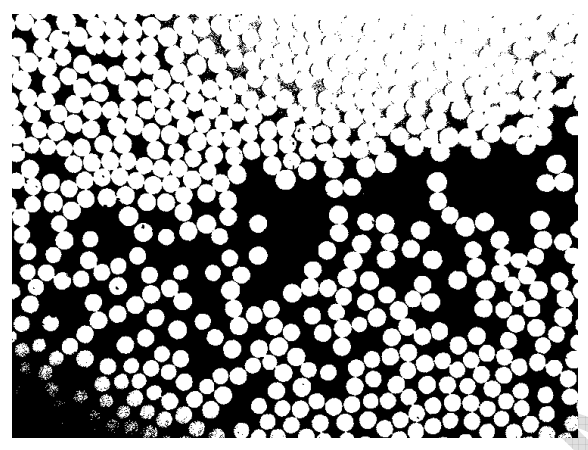

(a)

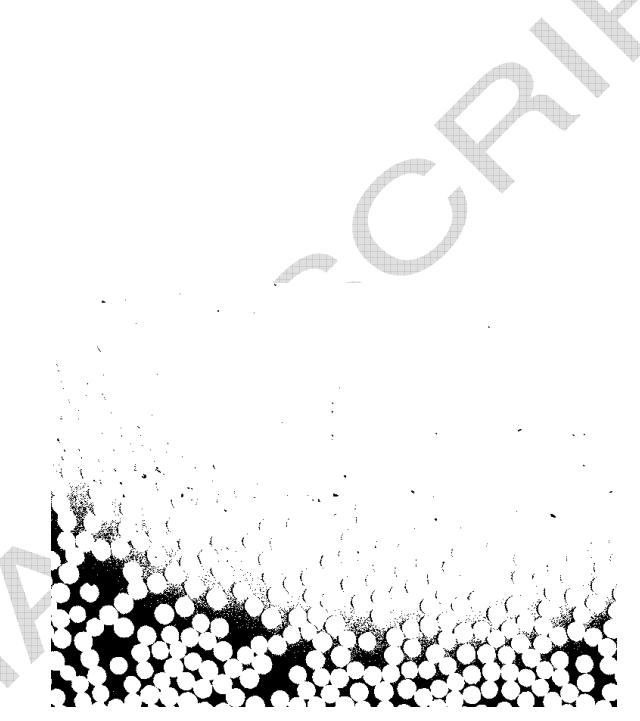

(b)

Fig. 3. Result of applying a binarization to the original image according to Otsu's algorithm [35]. (a) Threshold is set to 0.65 . Notice that it produces a poor segmentation at the top right and bottom-left corners. (b) Result of applying a 0.69 threshold to correctly segment the bottom-left fibers. It can be observed how a small change in the threshold produces a big change in the segmentation. This means that the selected threshold is located at a very unstable point. 


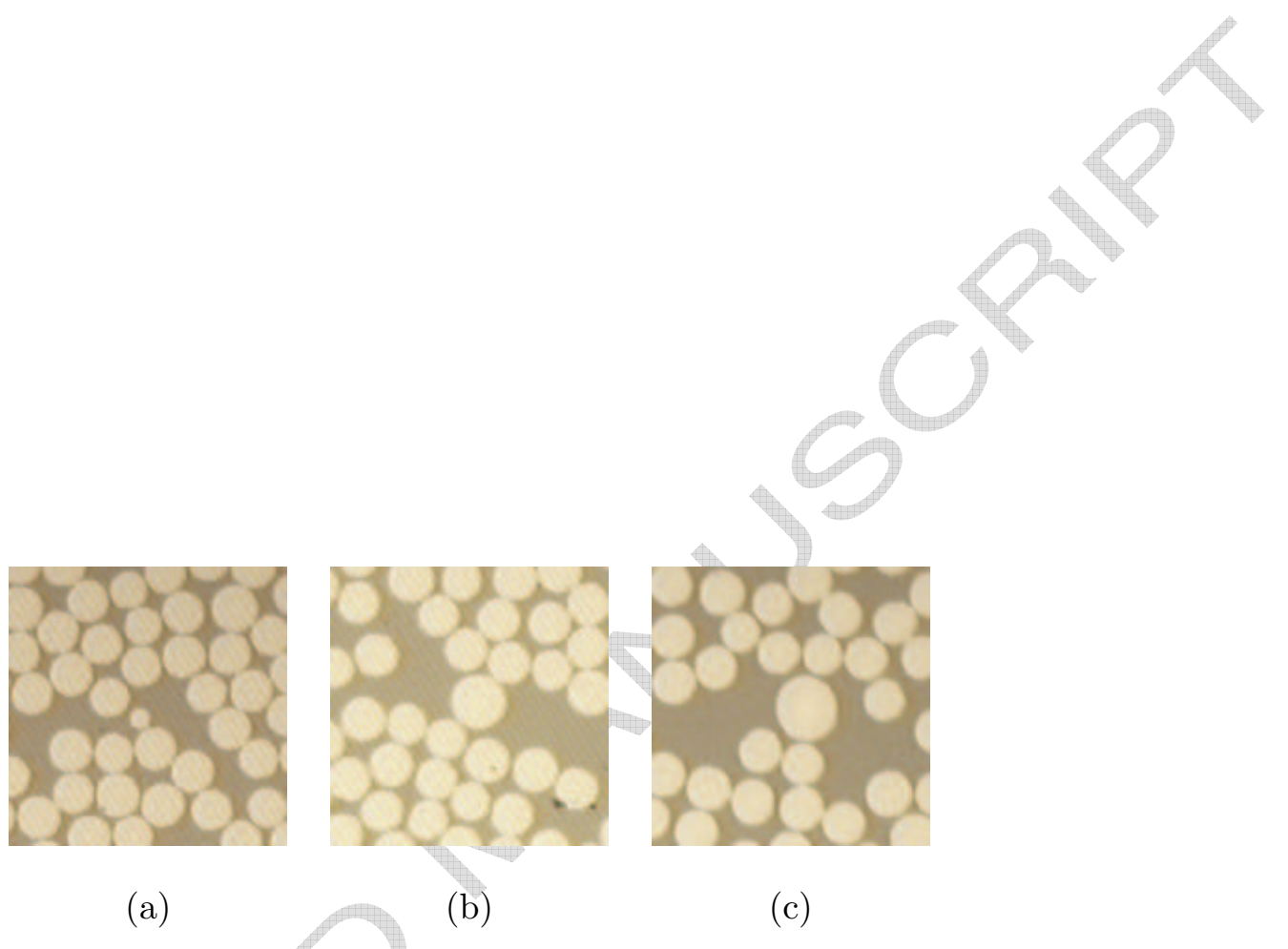

Fig. 4. Detail of 3 images showing fibers of different radii.

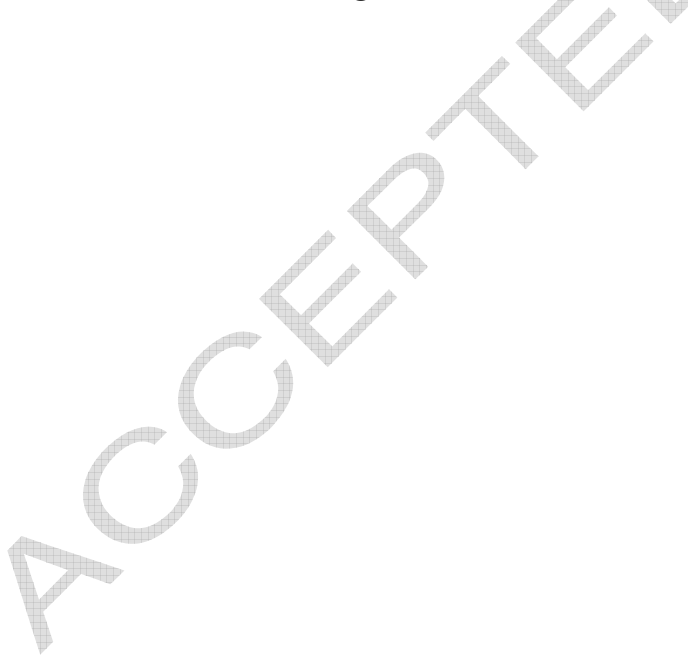




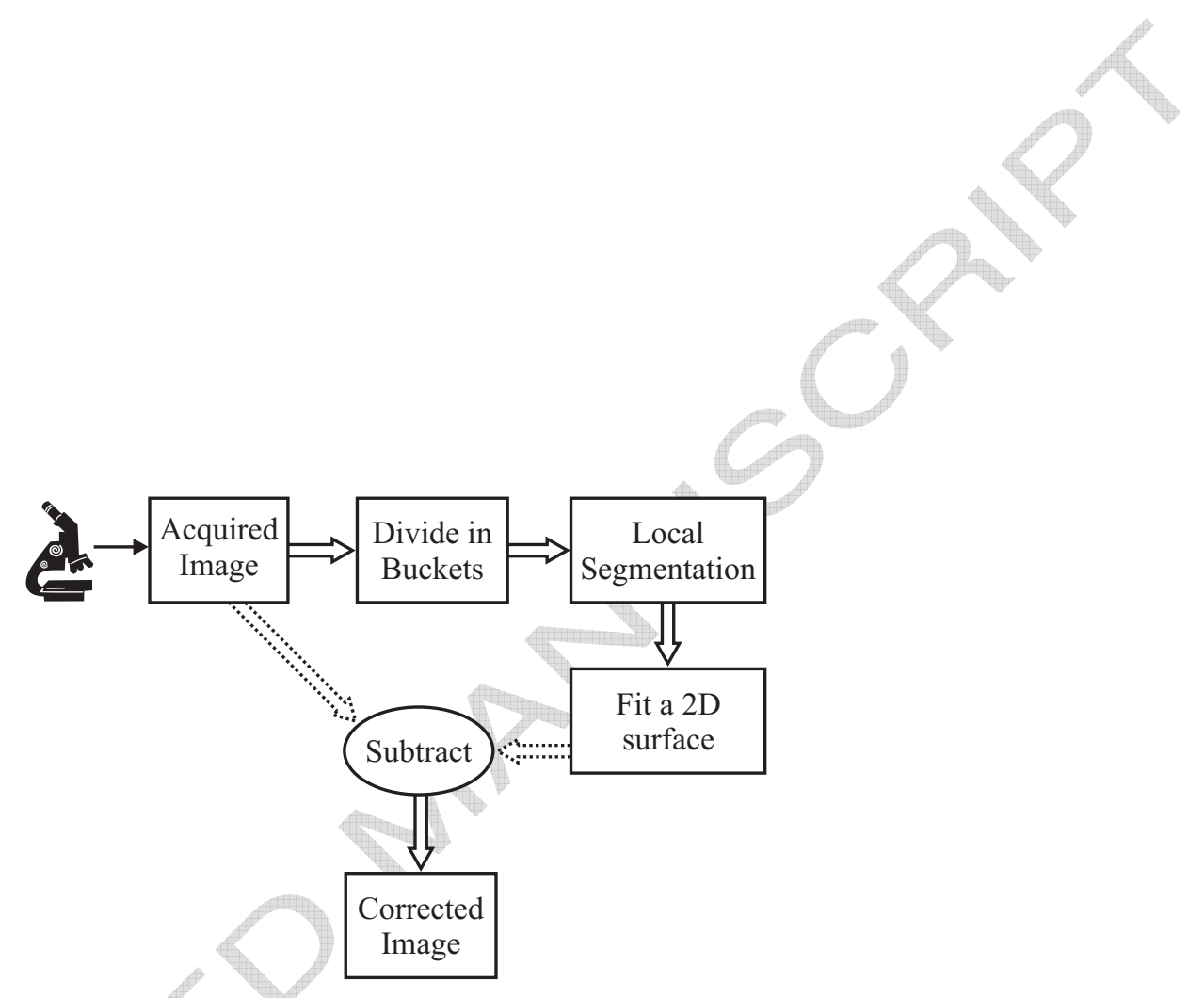

Fig. 5. Bloc diagram of the radiometric correction procedure. 


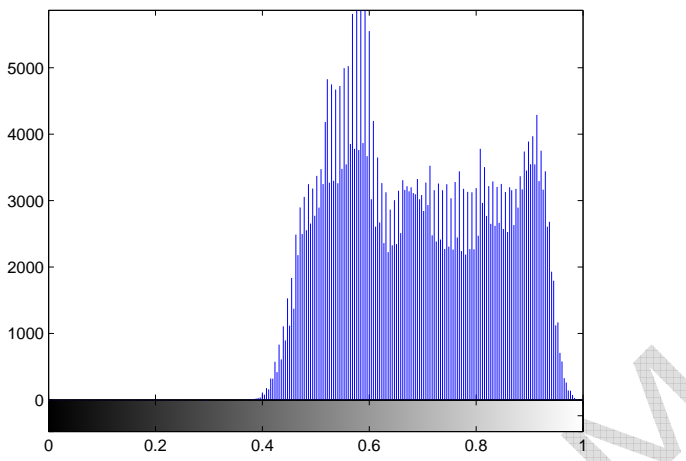

(a)

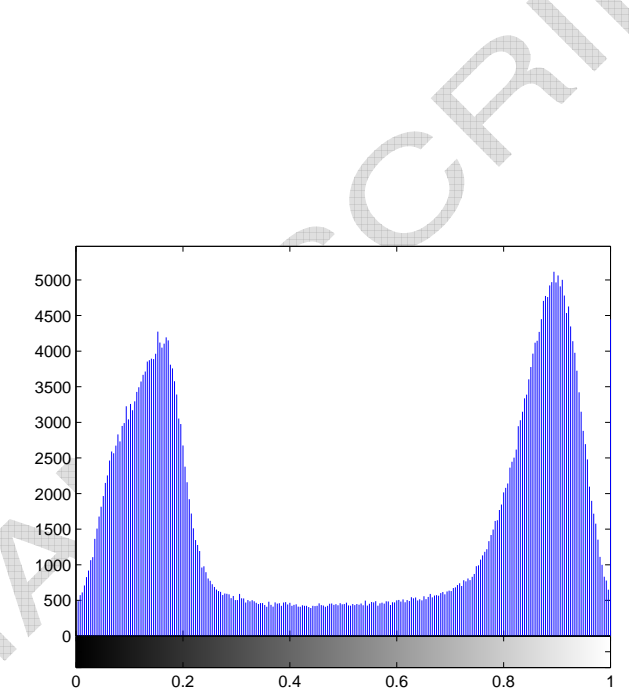

(b)

Fig. 6. Histograms of (a) the original image illustrated in Figure 1, and (b) the image obtained after radiometric correction. In (a) it is impossible to find an adequate threshold, as illustrated in Figure 3. The (b) histogram is now well conditioned to binarize the image applying Otsu's algorithm. 


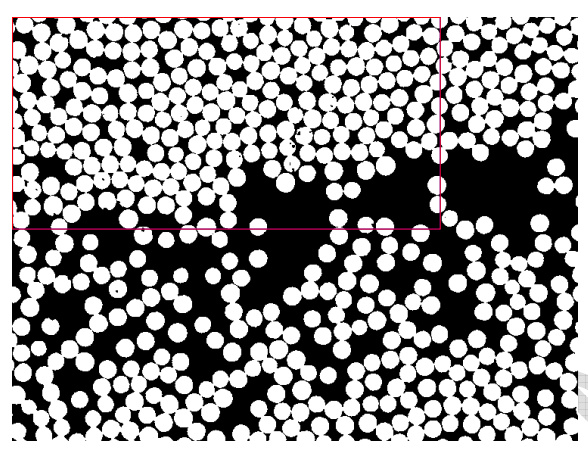

(a)

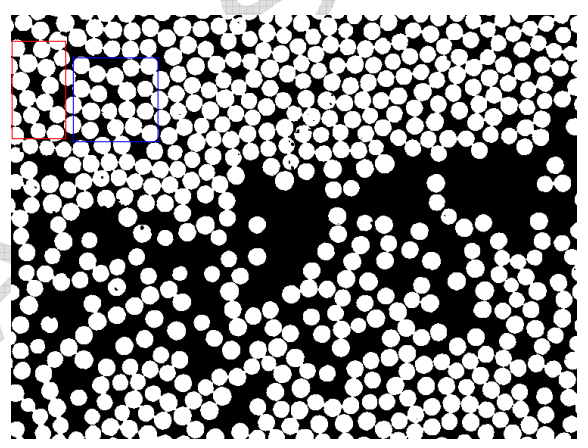

(b)

Fig. 7. Binarization of the radiometrically corrected image. (a) The red rectangle frames the largest object after applying a Connected Component Labeling (CCL) strategy. The largest object defined in (a) is eroded, giving rise to image (b). Now the objects with maximum height (red) and width (blue) are framed. 


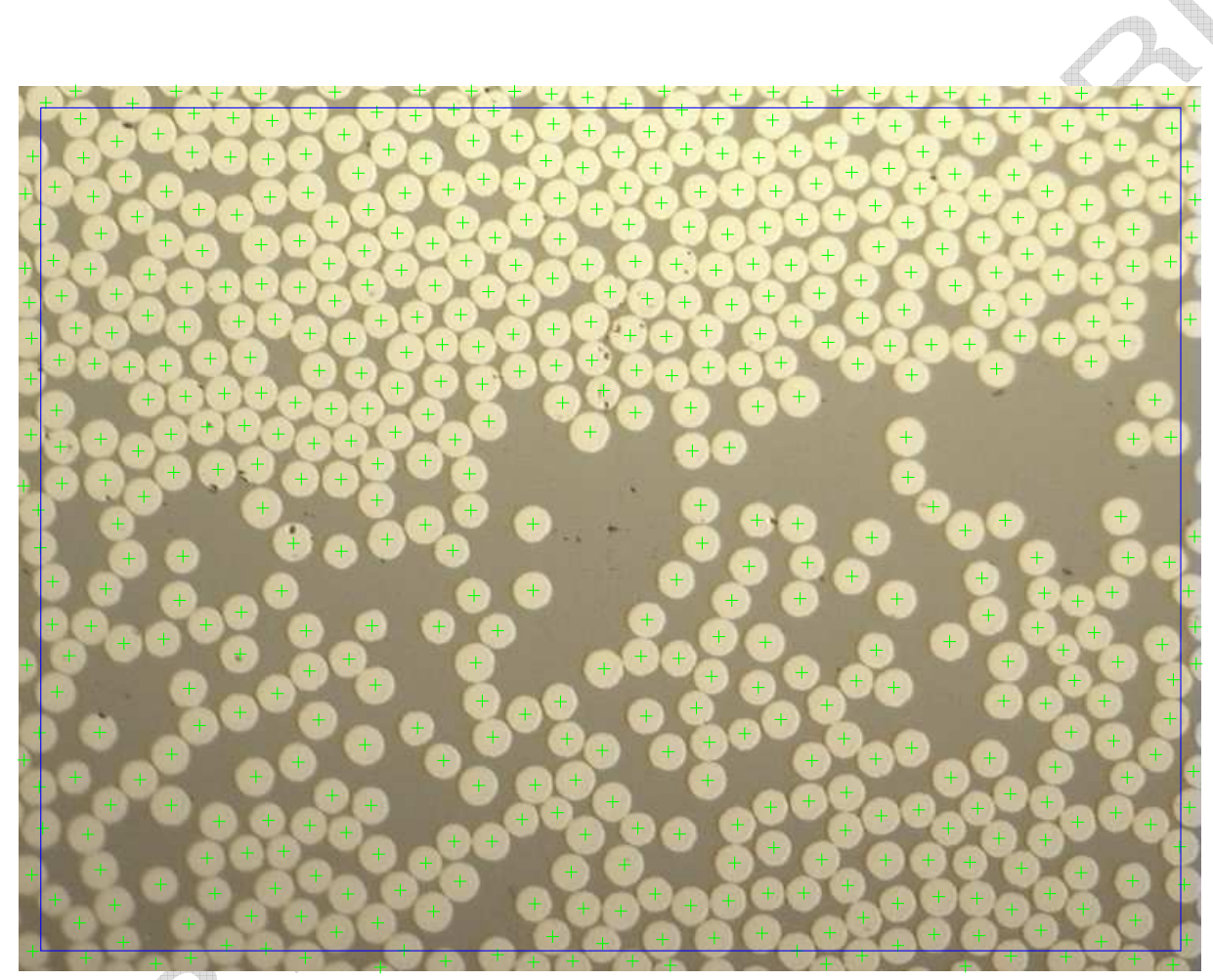

Fig. 8. Localization of the automatically detected fibers. Due to the nature of the algorithm, it can be observed how the algorithm fails to correctly locate the center of the fibers when they are touching the border of the image. For this reason, only the fibers having their gravity center within the rectangle are considered.

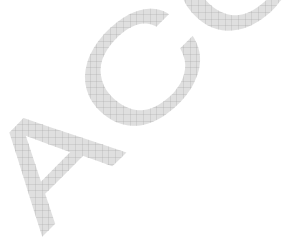




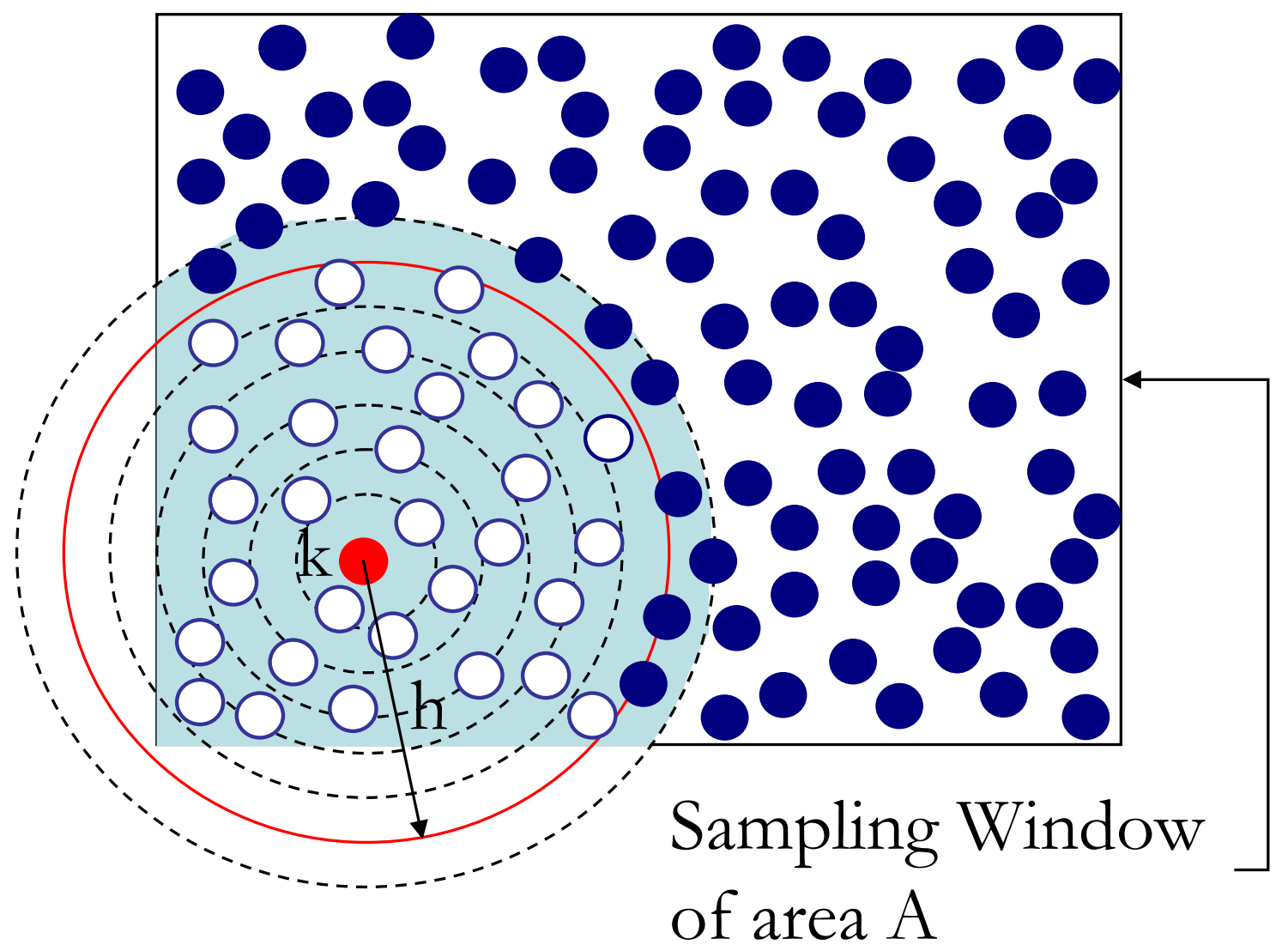

Fig. 9. Estimation of Ripley's K-function, $K(h)$. 

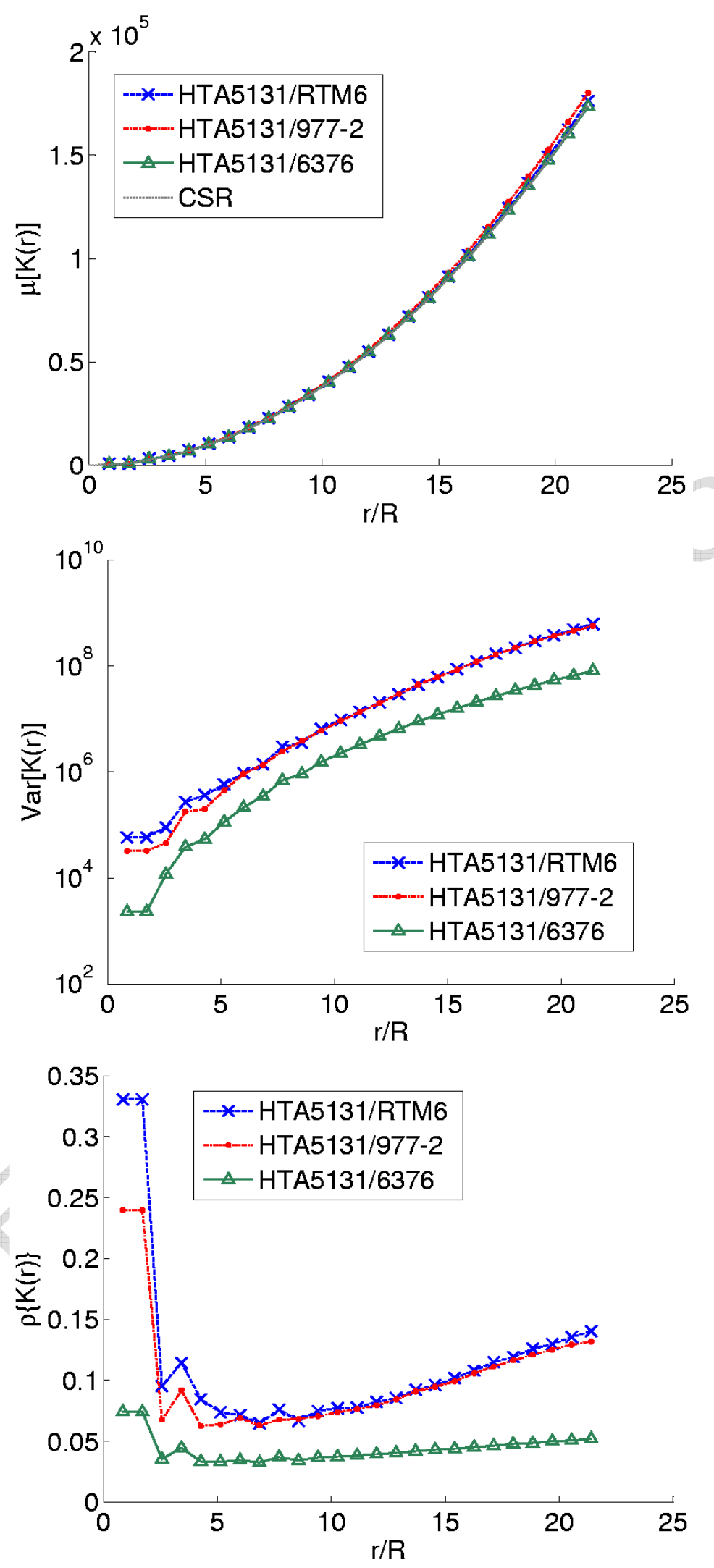

Fig. 10. Mean(top), variance(middle) and coefficient of variation (bottom) of $K(h)$ for the analyzed materials 

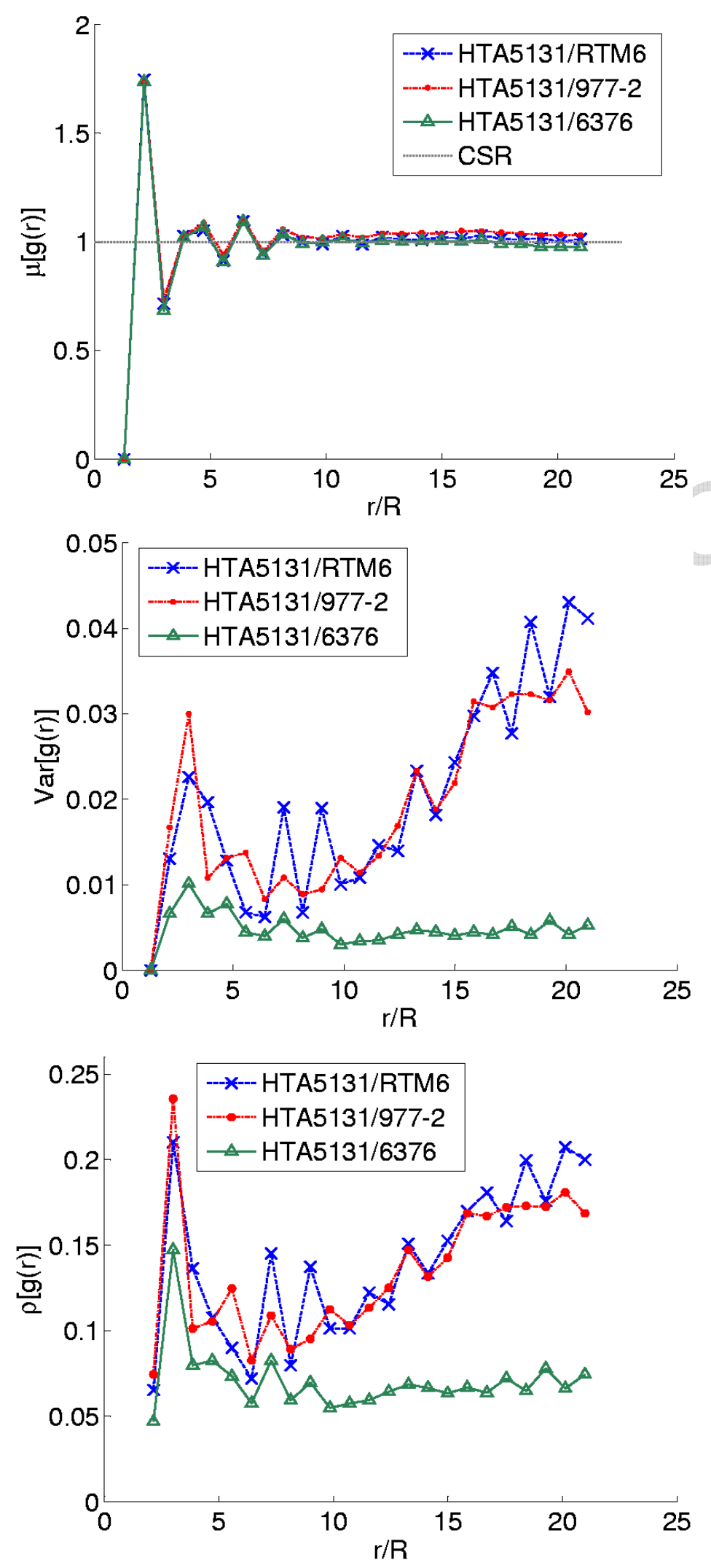

Fig. 11. Mean(top), variance(middle) and coefficient of variation (bottom) of $g(h)$ for the analyzed materials 


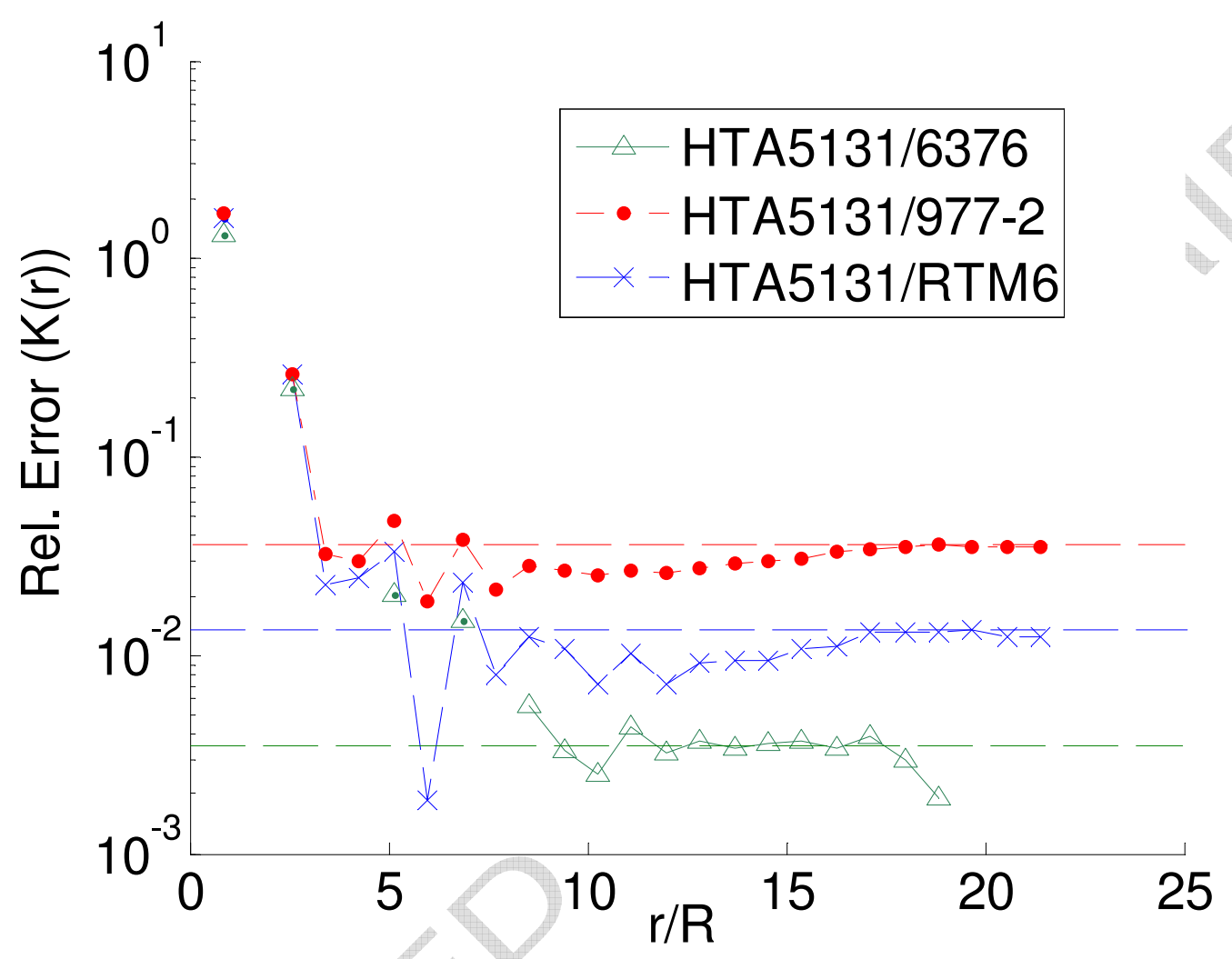

Fig. 12. Relative error for $K(h)$ 


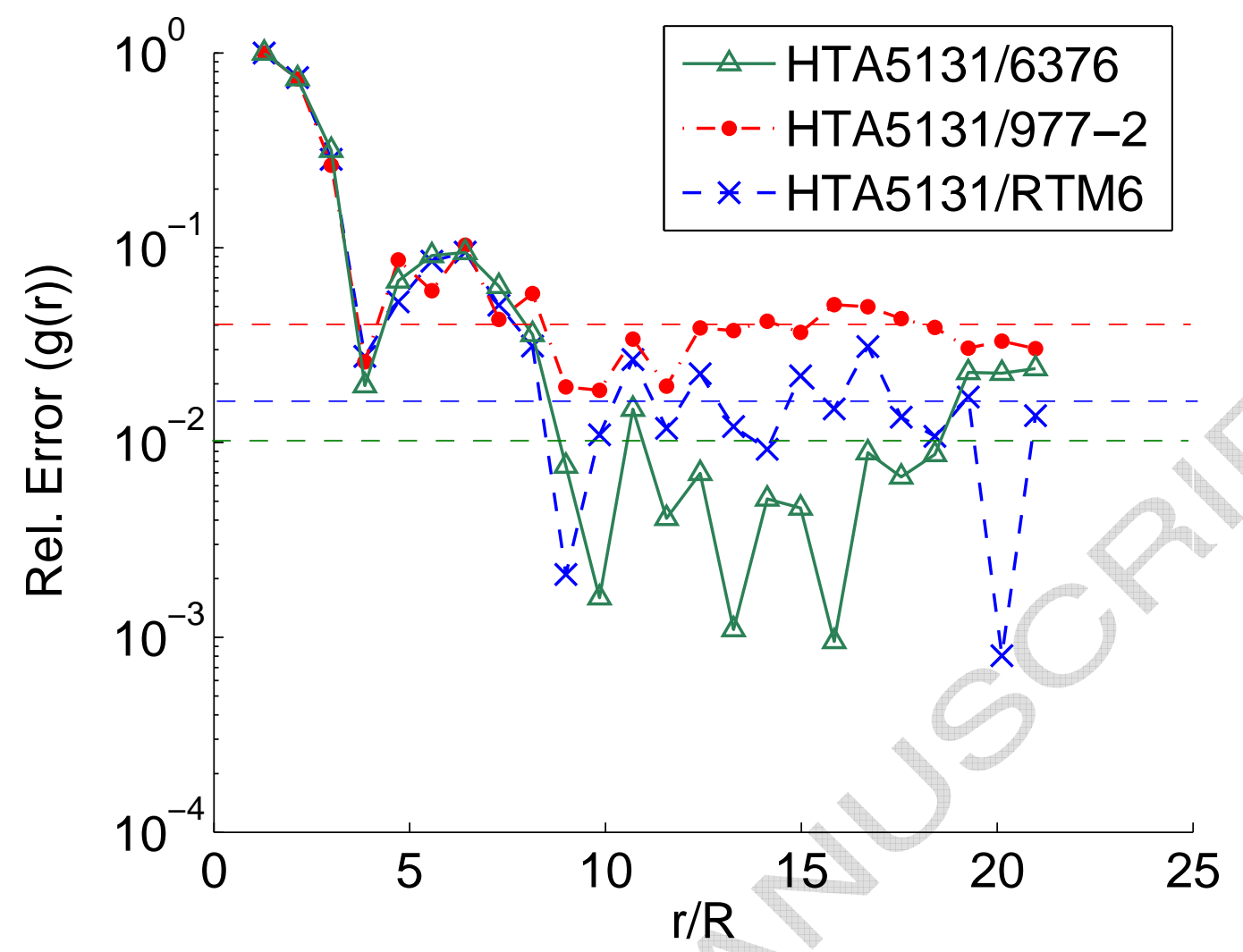

Fig. 13. Relative error for $g(h)$ 


\section{Tables}

Table 1

Elastic properties of the fiber (HTA5131) and the three analyzed matrixes. $\left(^{*}\right)$ Transverse modulus 


\begin{tabular}{|ccc|}
\hline Material & $\mu\left(\varepsilon^{u}\right)$ & $\sigma\left(\varepsilon^{u}\right)$ \\
\hline HTA5131/RTM6 & 0.0034 & 0.0063 \\
HTA5131/977-2 & 0.0112 & 0.0099 \\
HTA5131/6376 & 0.0081 & 0.0009 \\
\hline
\end{tabular}

Table 2

Ultimate strain statistics for three different CFRPs 


\begin{tabular}{|cccc|}
\hline Material & $\mu\left(v_{f}\right)$ & $\sigma^{2}\left(v_{f}\right)$ & $\rho\left(v_{f}\right)$ \\
\hline HTA5131/RTM6 & 0.5771 & 0.002383 & 0.08458 \\
HTA5131/977-2 & 0.5725 & 0.002118 & 0.08037 \\
HTA5131/6376 & 0.6052 & 0.0004436 & 0.03480 \\
\hline
\end{tabular}

Table 3

Volume fraction statistics computed for three different CFRPs 